\title{
Review of: "Experimental study on the explosion characteristics of hydrogen-methane premixed gas in complex pipe networks"
}

chunmiao yuan ${ }^{1}$

1 Northeastern University

Potential competing interests: The author(s) declared that no potential competing interests exist.

This is meaningful research in the field of gas explosion and prevention. The paper helps to understand the law of flame propagation and overpressure in a complex pipe network. Here are some minor comments: 1. I noticed that the authors had conducted three tests for each group. It could be better to add an error bar in Figures 3, 6, and 8.

2. The explosion characteristics are highly related to the uniform of premixed gas. Although a pump has been used for gas circulation, specific values of gas concentration are suggested provided.

3. In Figure 4, the B-Spline seems not appropriate to indicate the variation of the $\mathrm{P}_{\max }$ of premixed gas. 